\title{
Deconstructing the abundance-suitability relationship in species distribution modelling
}

\author{
Alberto Jiménez-Valverde ${ }^{1}$ Pedro Aragón ${ }^{2,3}$ Jorge M. Lobo ${ }^{2}$ \\ 'Departamento de Ciencias de la Vida, Facultad de Ciencias, Universidad de Alcalá (UAH), Madrid, Spain \\ ${ }^{2}$ Departamento de Biogeografía y Cambio Global, Museo Nacional de Ciencias Naturales (MNCN), Madrid, Spain \\ ${ }^{3}$ Departamento de Biodiversidad, Ecología y Evolución, Facultad de Ciencias Biológicas, Universidad Complutense de Madrid (UCM), Madrid, Spain .
}

\section{Correspondence}

Alberto Jiménez-Valverde, Departamento de Ciencias de la Vida, Facultad de Ciencias, Universidad de Alcalá (UAH), E-28871 Alcalá de Henares, Madrid, Spain. Email: alberto.jimenezv@uah.es

Funding information

Ministerio de Economía y Competitividad, Grant/Award Number: CGL2014-56416-P, CGL2017-89000-P, RYC-2011-07670 and RYC-2013-14441

\begin{abstract}
Aim: Local suitability estimated with species distribution models (SDMs) could indicate the maximum abundance attainable by species. Often the abundance-suitability relationship is wedge-shaped because species do not reach their maximum potential in every suitable location. We explore how SDM performance, the amount of information lost when converting continuous abundance into presence-absence data and species prevalence influence the abundance-suitability relationship.
\end{abstract}

Location: Undefined.

Time period: Undefined.

Major taxa: Virtual species.

Methods: Different abundance scenarios were simulated and converted into presence-absence maps. SDMs were parameterized using simulated predictors with different explanatory capacities. Quantile regressions were performed to characterize the abundance-suitability relationship. The strength of the wedge-shaped pattern was estimated using the difference in slopes between the 90th and 50th quantile lines, the goodness-of-fit of the 90th quantile regressions was calculated, and variation of both parameters was analysed as a function of SDM performance, prevalence and maximum abundance.

Results: The degree of wedge shape was directly related to maximum abundance. It also depended on SDM quality; the higher the discrimination capacity, the less wedge-shaped the abundance-suitability relationship and higher the goodness-of-fit of the 90th quantile regressions. Prevalence exerted a significant effect on the shape of the relationship by influencing - together with maximum abundance - the extent of information loss.

Main conclusions: SDMs cannot predict actual abundance even when the true determinants of abundance are included as predictors. Because presence-absence and abundance data are related but are different variables, wedge-shaped patterns are unavoidable. Discrimination capacity and prevalence affect the strength of the wedge-shaped pattern, so understanding their effects is necessary before any biological explanation is provided 
for the abundance-suitability relationship. Suitability maps derived from SDMs may transmit a false sense of precision at a local scale and should not be used as a perfect surrogate when abundance information is required.

\section{KEYWORDS}

abundance, discrimination capacity, heteroscedasticity, occurrence probability, presence- absence models, prevalence

\section{INTRODUCTION}

For decades, understanding and predicting patterns of species occurrence (presence-absence) and abundance across space and time have formed the cornerstone of ecology and biogeography since it is assumed that the effects of the environment and evolutionary history on species are directly reflected in these patterns (Brown et al., 1995, 1996). Abundance is essentially the product of demographic processes (reproduction, survival, growth) and dispersal dynamics, which are evolutionarily shaped traits that are modulated by environmental factors and biotic interactions (Lowe \& McPeek, 2014). Occurrence patterns may be considered the unbalanced binarization of 'real' and generally unknown abundance gradients (no. individuals $=0$ equals absence; no. individuals $\geq 1$ equals presence). Presence-absence maps are thus simplifications of real patterns found in nature because we are unable to collect exhaustive data on population abundance variations across broad regions (Kissling et al., 2018). Significant changes in abundance can occur without affecting the presence of the species (Ashcroft et al., 2017). Thus, assessing spatio-temporal dynamics in abundance rather than in occurrence is the preferred means to detect future extinctions of declining populations or probable expansions of, for instance, exotic species or disease vectors (Ehrlén \& Morris, 2015). Additionally, due to demographic stochasticity, abundance is thought to be directly related to the probability of persistence (O'Grady et al., 2004; Pimm et al., 1988; Soininen \& Heino, 2005), which is a highly valuable criterion for conservation success (Williams \& Araújo, 2000). Abundance may also indicate the impact of species on the environment, which is of importance in the case of invasive species and disease vectors (Parham et al., 2015; Parker et al., 1999).

Despite the evident advantages of using abundance data for basic and applied purposes, such information is almost non-existent for most species across significant parts of their geographical ranges (but see attempts in Salguero-Gómez et al., 2016; Santini et al., 2018; Thibault et al., 2011). In contrast, occurrence data are much more easily obtainable, rendering the generation of distribution maps for many species in most parts of the world a relatively easy and inexpensive task (Acevedo et al., 2016). Occurrence information is already being used to tackle applied research questions (e.g. Guisan et al., 2013; Jiménez-Valverde et al., 2010, 2011; Peterson et al., 2011). Thus, there is evident interest in using presence-absence maps to predict abundance 
(Kunin, 1998). Total regional abundance can be reasonably estimated for small spatial extents using occurrence maps (Ashcroft et al., 2017; Yin \& He, 2014; and references therein). However, these procedures cannot be used to estimate local variations in abundance; furthermore, they require reliable presence-absence data and their predictions are not transferable to other temporal or spatial scenarios. One way to overcome these drawbacks might be to use species distribution models (SDMs) in which occurrence data for the focus species are linked to specific ecogeographical predictors via a statistical or logical function (Franklin, 2009; Guisan et al., 2017; Peterson et al., 2011).

The output of SDMs takes the form of a continuous variable that reflects the probability of presence sensu stricto or a relative degree of suitability depending on the modelling technique and the type of distributional data used (Phillips et al., 2006; Real et al., 2006). For the sake of brevity, we will use the general term 'suitability' to refer to SDM output. In general, according to the Hutchinsonian (Hutchinson, 1957, 1978) and Grinellian (Grinnell, 1917) niche concepts (see Soberón, 2007), suitability and local abundance should be positively correlated since population densities should be highest where optimum environmental conditions occur and lower towards the periphery of the ecological niche (Brown et al., 1995; Hengeveld, 1990; Martínez-Meyer et al., 2012; Osorio-Olvera et al., 2020). However, this does not always seem to be the case (Dallas \& Hastings, 2018; Dallas et al., 2017; but see Osorio-Olvera et al., 2020 and references therein), and when it is, the effect size is not usually very high (see review in Weber et al., 2016).

The first published studies assumed a simple linear relationship between suitability and local abundance (e.g. Jiménez-Valverde, Diniz, et al., 2009; Nielsen et al., 2005; Pearce \& Ferrier, 2001), but VanDerWal et al. (2009) realized that the relationship was in fact triangular or wedge-shaped, that is, low suitability values entail low abundance values, but high suitability scores could involve either high or low abundance values. Many others have corroborated this wedge-shaped relationship between suitability and abundance (e.g. Acevedo et al., 2017; Carrascal et al., 2015, 2017; Gutiérrez et al., 2013; Lunghi et al., 2018; Muñoz et al., 2015; Tôrres et al., 2012), suggesting that suitability may indicate the maximum potential abundance that a species could attain in a given place (Acevedo et al., 2017; Muñoz et al., 2015; VanDerWal et al., 2009). In general, wedge-shaped relationships are often caused by a predictor variable that restricts the upper limit of a response variable but cannot determine its precise value because of other unknown covariates (Thomson et al., 1996). Dispersal constraints or non-considered biotic and abiotic factors have been cited to explain the finding that a species may attain low abundance values in places estimated as suitable, that is, a wedge-shaped pattern in the abundance-suitability relationship (VanDerWal et al., 2009; Weber et al., 2016). Thus, as suggested by Jiménez-Valverde (2011), the strength and shape of the abundance-suitability relationship depends—at least to a certain extent - on the discrimination capacity of the predictors included in the SDMs, although other authors 
disagree (e.g. Guarino et al., 2012).

Alternatively, the wedge-shaped pattern may simply be explained by the loss of information that occurs when abundance is converted into presence-absence data. This information loss is crisply illustrated by Bahn and McGill (2013), who explained that if a species is present in exactly half of the study region, then the probability of correctly predicting occurrence in a given territorial unit is .5; however, there is a zero probability of correctly estimating abundance. Lastly, the prevalence (the ratio of presence to total number of presences and absences) of the target species may also be a decisive factor to explain the wedge-shaped pattern of the relationship between abundance and suitability. Species prevalence is known to exert pervasive effects on SDM results (Fukuda \& De Baets, 2016; Jiménez-Valverde, Lobo, et al., 2009; Santika, 2011; Somodi et al., 2017) and the influence of unbalanced prevalence on the abundance-suitability relationship remains entirely unexplored.

In this study, virtual species were used to assess how the abundance-suitability relationship is affected by (a) the predictive quality of SDMs, (b) the amount of information lost when converting continuous abundance into binary presence-absence data, and (c) species prevalence. Virtual species were used to assure a broad range of scenarios with different prevalence and maximum abundance values, rendering it possible to generate SDMs with varying degrees of performance and to guarantee that the data (occurrence and abundance) were free of error (no misidentifications, unreliable records or biased data). Virtual species and occurrence data were generated as simply as possible, without considering the role of other possible causes influencing the wedge-shaped abundance-suitability relationship, such as dispersal limitations or interactions with other species (VanDerWal et al., 2009), because we wanted to determine if the relationship was evident even in the absence of these processes.

\section{METHODS}

\subsection{Abundance and presence-absence simulations: the virtual species}

A digital elevation model (GTOPO30; https://www.usgs.gov/centers/eros) in raster format $(100 \times 100$ pixels $)$ for the geographical area in North America between $48^{\circ} 12^{\prime} 28.8^{\prime \prime}-52^{\circ} 22^{\prime} 12^{\prime \prime} \mathrm{N}$ and $116^{\circ} 15^{\prime} 0^{\prime \prime}-120^{\circ} 24^{\prime} 0^{\prime \prime} \mathrm{W}$ (mean $=1,395.8 \mathrm{~m}$ a.s.1., median $=1,385$, first quartile $=1,002$, third quartile $=1767$, min. $=294, \max .=3,086$; see Supporting Information Figure S1.1) was used to simulate abundance $(N)$ as follows. $N$ was only conditioned on the variable elevation $(x)$ following the Gaussian (symmetric bell-shaped) function $N=c \cdot e$ $\left(-x-u^{2} / 2 t^{2}\right)$, where $c$ is the maximum possible value of $N, u$ is the optimum (the value of $x$ where $N$ is maximum), and $t$ is the species' tolerance (width). $N$ values were converted to integers and were subsequently converted to presence-absence assuming a uniform detection probability across the entire area, that is, $N>0$ equals presence while $N=0$ equals absence. 


\subsection{Species distribution models}

Generalized additive models (GAMs) with a logit link function (binomial family) were used to fit SDMs. The 10,000 cells were randomly divided into two halves — a training and a testing dataset — of 5,000 cells each. Each time an SDM was fitted, noise was added to elevation (see next section 2.3), and a GAM with penalized thin plate regression splines (Wood, 2003) was fitted, setting the maximum complexity dimension of the smooth $(k)$ to 4 . The smoothing parameter estimation problem was solved using the generalized cross-validation method for an unknown scale parameter (Wood, 2004). To reduce over-fitting of the splines to the data without evident degradation in prediction error performance, a penalty per degree of freedom was introduced in the generalized cross-validation criterion by setting the gamma parameter to 1.4, which forced models to be smoother than they might otherwise be (Kim \& Gu, 2004). For more details on GAM procedures, see Wood (2017). GAMs were run using the mgcv package version 1.8-24 (Wood, 2017) in R version 3.1.1 (R Development Core Team, 2014).

Model discrimination capacity was assessed on the testing data-set after applying a threshold to continuous suitability values. We used the threshold of equivalency, which renders sensitivity (true positive rate) equal to specificity (true negative rate) for this purpose because the derived statistic $S e^{*}$ is a direct and more appropriate measure of discrimination than the area under the receiver operating characteristic (ROC) curve (AUC) (Jiménez-Valverde, 2012, 2014). Yet, because the AUC is the most widely used discrimination statistic (despite its shortcomings; see Lobo et al., 2008), we also estimated it to ensure that the overall findings were robust to the discrimination measure. All calculations were performed using ROCR package version 1.0-7 (Sing et al., 2005) for R.

\subsection{Adding noise to the predictor}

A set of 100 random fields with varying degrees of spatial autocorrelation was generated via unconditional Gaussian simulation using the gstat package version 2.0-2 (Gräler et al., 2016) for R. The gstat function was employed to simulate the fields using simple kriging by arbitrarily setting the beta parameter (kriging mean) to 1 and nmax (number of nearest observations used for kriging simulation) to 20. The variogram model was exponential, the psill parameter (still) was arbitrarily set to 0.025 and the range parameter was allowed to randomly vary between 2 and 30 (in increments of 4) to change the degree of spatial autocorrelation. Each time a GAM was fitted (see previous section 2.2), some noise was added to the predictor by multiplying one randomly chosen field by a random number between 1 and three times the maximum value of elevation (this range of values was chosen by trial and error to assure a broad range, and avoid a highly skewed distribution, of discrimination values) and adding this to the variable elevation. This noise was added to the predictor in order 
to randomly vary the discrimination capacity of the SDMs.

\subsection{Abundance-suitability relationship}

$N$ values in each of the 5,000 cells of the testing dataset were regressed onto the suitability values predicted by GAMs $(P)$ by means of linear quantile regression models fitted to the 50th and 90th percentiles (see Cade \& Noon, 2003 and references therein). Previously, $P$ values were converted to $P^{*}$ values using the expression $-\ln$ (1 - P) because (a) it tends to linearize the relationship between $P$ and $N$ (a requisite for linear quantile regressions) and (b) it has been shown to successfully convert probabilities of occurrence into either local or regional species densities (Carrascal et al., 2015; Gerrard \& Chiang, 1970; Gerrard \& Cook, 1972). The strength of the wedge-shaped pattern in the relationship between $N$ and $P^{*}$ was assessed by means of the difference in the slopes between the 90th and 50th percentiles $(D)$. Large differences in slopes denote complex interactions between $N$ and $P^{*}$, thus showing wedge-shaped patterns instead of clear linear relationships. We

estimated $R^{1}$ for the 90th quantile regression model (hereafter $R$ ) as a goodness-of-fit measure (Koenker \& Machado, 1999). Only closeto-zero $D$ values together with high $R$ values indicate that $P *$ is a good predictor of $N$. Linear quantile regressions were fitted applying the modified version of the Barrodale and Roberts algorithm using the quantreg package version 5.36 (Koenker, 2018) for R.

\subsection{Experiments}

\subsubsection{Experiment \#1: fixed maximum abundance}

In experiment \#1, maximum possible abundance was set at 500. This was controlled by the $c$ parameter of the Gaussian function (see section 2.1) and represents the amount of information that is lost when abundance is converted into presence-absence data; the higher the maximum abundance, the greater the information loss. A total of 20,000 iterations were run, and in each iteration a random value between the minimum and maximum values of elevation was assigned to $u$, and a random value between 10 and 360 was assigned to $t$, thus changing the species' prevalence (this range for $t$ was chosen by trial and error to enssure an approximately uniform distribution of prevalence values). Abundance and presence-absence surfaces were simulated (see section 2.1). The 10,000 cells were randomly divided in two, and a GAM was fitted using one half, while $S e^{*}$ and $D$ were calculated using the other half (see sections 2.2 and 2.3). Of the 20,000 iterations, those that generated virtual species with very unbalanced prevalences $(<.01$ or $>.99)$ were discarded to assure a sufficiently large sample size for model training and evaluation (Jiménez-Valverde, 2020; Jiménez-Valverde, Lobo, et al., 2009). In addition, iterations that generated testing datasets in which maximum abundance $(\max (N)) \neq 500$ were also discarded, as were iterations with $S e^{*}$ values of $<.55$ or $>.95$ because these models perform either 
unrealistically bad or good, respectively. By the end, 15,734 iterations remained for further analyses.

\subsubsection{Experiment \#2: fixed discrimination capacity}

In this experiment, 70,000 iterations were run. In each iteration, a random value between 5 and 1,000 was assigned to $c$, a random value between the minimum and maximum values of elevation was assigned to $u$, and a random value between 70 and 360 was assigned to $t$ (this range for $t$ was chosen by trial and error to ensure an approximately uniform distribution of prevalence values). SDMs were built as in experiment \#1 and again, of the 70,000 iterations, those that generated virtual species with very unbalanced prevalences were deleted. In this case, iterations that generated $\mathrm{Se}^{*}$ values of $<.70$ or $>.80$ (equivalent to AUC values of $<.74$ or $>.91$; see Jiménez-Valverde, 2014) were also discarded. By the end, 17,475 iterations remained for further analyses in this case.

The $\mathrm{R}$ code and necessary files for running the two experiments are provided in Supporting Information Appendix S2.

\subsection{Analysis of the results}

In experiment $\# 1$, outliers of $D$ were eliminated. The relationship between $S e^{*}$ (or AUC) and $D$, and the influence of prevalence, was explored using dot plots and 2-D surface plots based on local polynomial regression analysis. The formula $D \sim S e^{*} \times$ prevalence (or $D \sim$ AUC $\times$ prevalence) was fitted by applying the loess function in $\mathrm{R}$ with 1-degree polynomial and a degree of smoothness (span) equal to 0.25 . The relationship between $S e^{*}$ (or AUC) and $R$, and the influence of prevalence, were explored in the same way.

In experiment $\# 2$, the relationship between the maximum $N$ value in the testing dataset $(\max (N))$ and $D$, and the influence of prevalence, were explored using dot plots and 2-D surface plots based on local polynomial regression analysis. The formula $D \sim \max (N) \times$ prevalence was fitted by applying the loess function as in experiment \#1.

\section{RESULTS}

\subsection{Experiment \#1: fixed maximum abundance}

In general, the relationship between $D$ and $S e^{*}$ was negative (Figure 1a) and that between $R$ and $S e^{*}$ was positive (Figure 2a); however, species prevalence exerted a clear effect on these patterns. In the case of very low prevalence values $(\leq .1)$, there was a positive relationship between $D$ and $S e^{*}$; low $S e^{*}$ values were associated with low $D$ values, whereas high $S e^{*}$ values were associated with either high or low $D$ values (Figure 1b). The relationship between $R$ and $S e^{*}$ was also positive, showing low $R$ values up to a certain 
medium $S e^{*}$ value, after which the variance of $R$ values increased (Figure $2 \mathrm{~b}$ ). In the prevalence range $(.1, .2]$, the relationship between $D$ and $S e^{*}$ started to reverse (Figure 1c), while the relationship between $R$ and $S e^{*}$ tended to linearize (Figure $2 \mathrm{c}$ ). From the prevalence range $(.2, .3]$, high $S e^{*}$ values were always associated with low $D$ values and high $R$ values, whereas low $S e^{*}$ values were associated with low $R$ values but either high or low $D$ values (Figures $1 \mathrm{~d}-\mathrm{k}$ and $2 \mathrm{~d}-\mathrm{k}$ ). As long as prevalence increased, the maximum value of $D$ decreased, even becoming negative at very high prevalence values (Figure $1 \mathrm{j}-\mathrm{k}$ ). Also, the relationship between $S e^{*}$ and $R$ tended towards an exponential shape (as was the case with very low prevalence values), showing low $R$ values up to a certain medium $S e^{*}$ value, after which the variance of $R$ values increased (Figure $2 \mathrm{j}-\mathrm{k}$ ). Overall, there was a unimodal relationship between prevalence and $D$, with a turning point around a prevalence value of .3 (Figure 11). Likewise, there was a general unimodal relationship between prevalence and $R$, with the highest $R$ values corresponding to highly discriminative models and prevalence values $<.5$ (Figure 21). The patterns using the AUC were virtually the same (Supporting Information Figure S1.2).

\subsection{Experiment \#2: fixed $S e^{*}(\geq .70$ and $\leq .80)$}

There was a clear positive effect of $\max (N)$ on $D$ : the higher its value, the higher the possible value of $D$ (Figure 3a). Once more, however, prevalence exerted an effect, conditioning the strength of the wedge-shaped pattern (Figure $3 \mathrm{~b}-\mathrm{k}$ ). The relationship between prevalence and $D$ was unimodal, with the turning point lo-There was a clear positive effect of $\max (N)$ on $D$ : the higher its cated around a prevalence value of 3 (Figure 31 ). $D$ values could value, the higher the possible value of $D$ (Figure $3 \mathrm{a}$ ). Once more, even be negative in the case of the highest prevalence values however, prevalence exerted an effect, conditioning the strength (Figure $3 \mathrm{k}$ ).

\section{DISCUSSION}

Our results clearly demonstrate that the wedge-shaped abundance-suitability relationship is a direct consequence of both the maximum abundance that a species may attain and the discrimination capacity of the SDM. Unavoidably, when maximum abundance is low, the strength of the wedge-shaped pattern cannot be high, independently of the discrimination power of the model. In the theoretical suitability relationship is a direct consequence of both the maximum case of a maximum abundance of one individual, there is no option abundance that a species may attain and the discrimination capacity for a wedge-shaped pattern since abundance and occurrence would be essentially the same variable. As maximum abundance increases, the wedge-shaped pattern becomes apparent and predicting abundance from suitability becomes more challenging (e.g. Carrascal et al., 2015). 

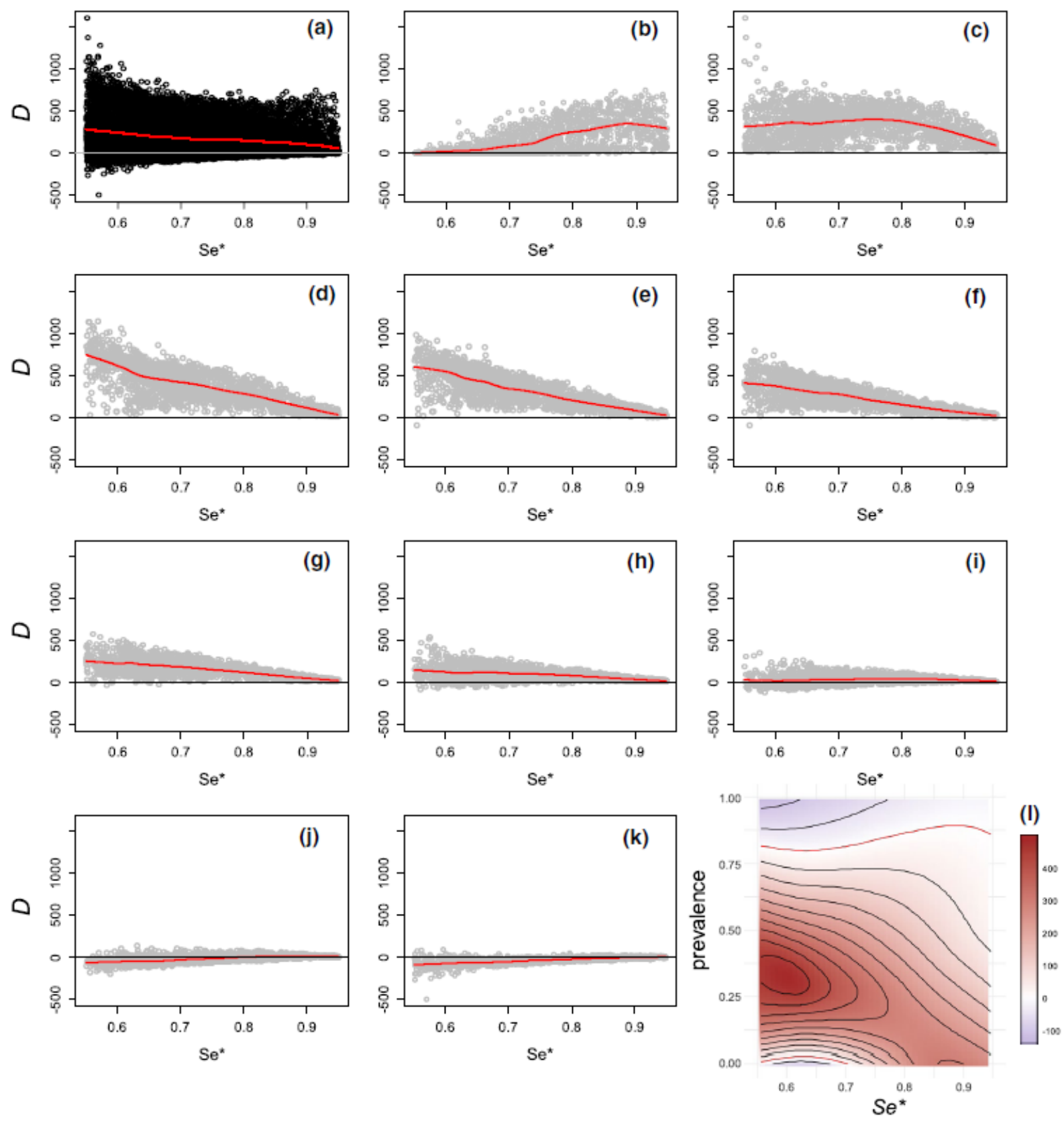

FIGURE 1 Results of experiment \#1 (fixed maximum abundance = 500). (a) Relationship between discrimination capacity $\left(S e^{*}\right)$ and strength of the wedge-shaped pattern $(D)$ considering all cases. In (b), the relationship between $S e^{*}$ and $D$ is shown only for cases with prevalence $\leq .1$; in (c), for cases with prevalence in $(.1, .2]$; in $(\mathrm{d})$, for cases with prevalence in $(.2, .3]$; in (e), for cases with prevalence in $(.3, .4]$; in (f), for cases with prevalence in $(.4, .5]$; in $(\mathrm{g})$, for cases with prevalence in $(.5, .6]$; in $(\mathrm{h})$, for cases with prevalence in $(.6$, $.7]$; in (i), for cases with prevalence in $(.7, .8]$; in $(\mathrm{j})$, for cases with prevalence in $(.8, .9]$; in $(\mathrm{k})$, for cases with prevalence $>$.9. The red line corresponds to the local polynomial regression fit (loess function in $\mathrm{R}$ with 1-degree polynomial and span $=0.25$ ). (1) Surface plot of $D$ as a function of $S e^{*}$ and prevalence. Warm colour indicates $D>0$, cool colour indicates $D<0$, white colour indicates $D=0$. Red contour line corresponds to the limit of $D=0$ 

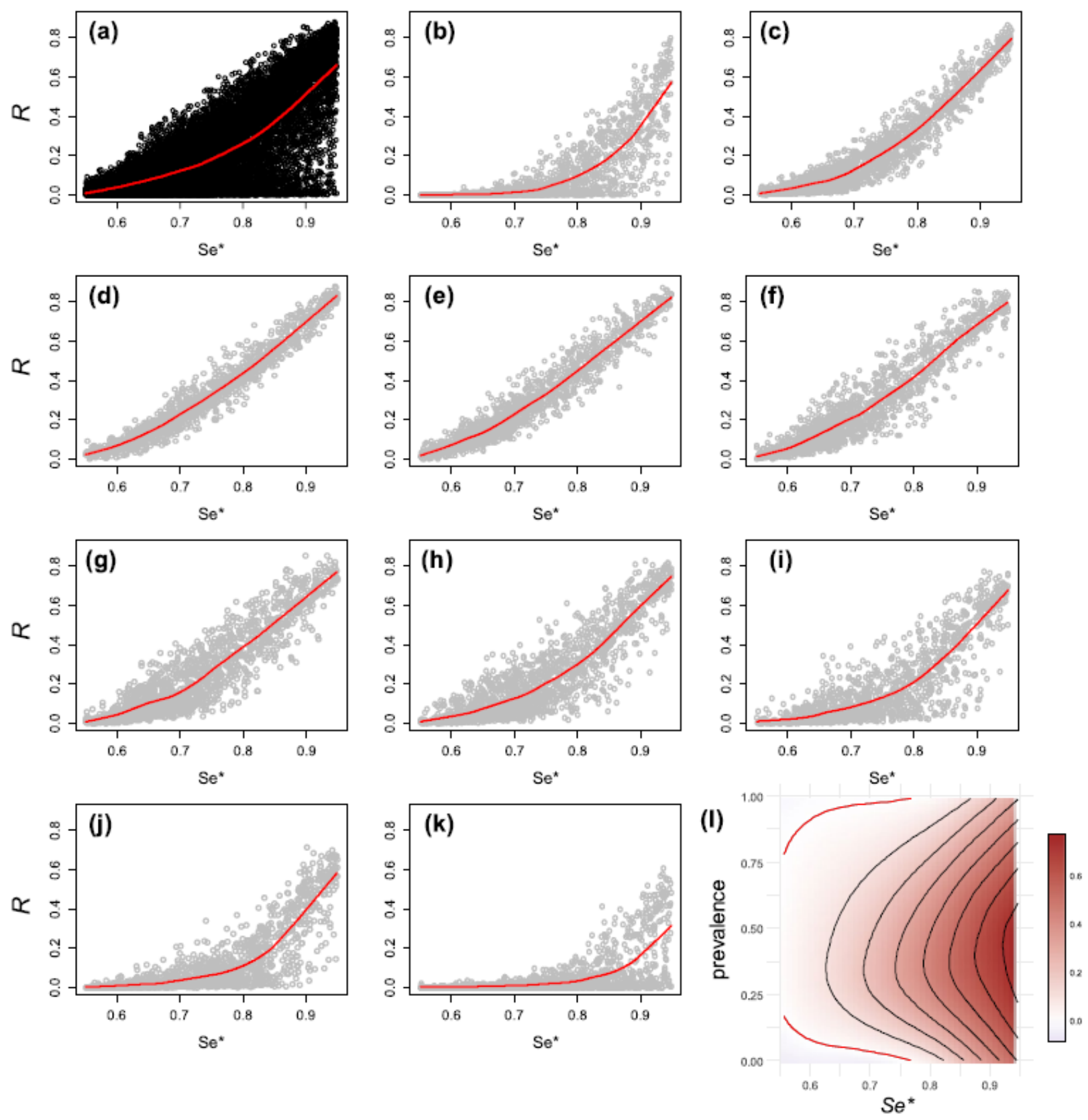

FIGURE 2 (a) Relationship between discrimination capacity $\left(\mathrm{Se}^{*}\right)$ and goodness-of-fit of the 90th quantile regression model $(R)$ considering all cases. In (b), the relationship between $S e^{*}$ and $R$ is shown only for cases with prevalence $\leq .1$; in $(\mathrm{c})$, for cases with prevalence in $(.1, .2]$; in $(\mathrm{d})$, for cases with prevalence in $(.2, .3]$; in (e), for cases with prevalence in $(.3, .4]$; in $(\mathrm{f})$, for cases with prevalence in $(.4, .5]$; in $(\mathrm{g})$, for cases with prevalence in $(.5, .6]$; in $(\mathrm{h})$, for cases with prevalence in $(.6, .7]$; in (i), for cases with prevalence in $(.7, .8]$; in (j), for cases with prevalence in $(.8, .9]$; in $(\mathrm{k})$, for cases with prevalence $>.9$. The red line corresponds to the local polynomial regression fit (loess function in $\mathrm{R}$ with 1-degree polynomial and $\mathrm{span}=0.25$ ). (1) Surface plot of $R$ as a function of $S e^{*}$ and prevalence. The warmer the colour, the higher $R$. Red contour line corresponds to $R=0$ 

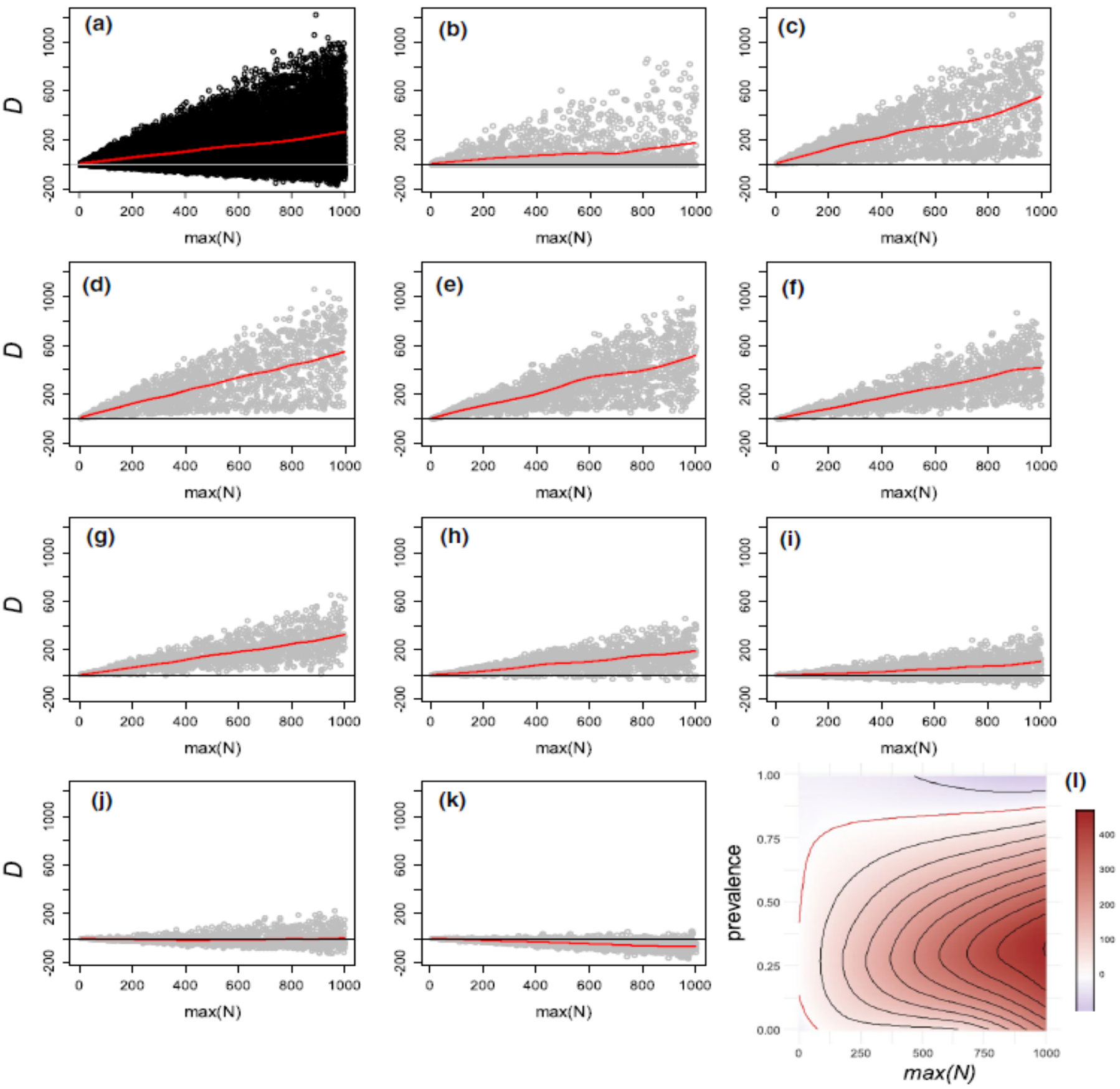

FIGURE 3 Results of experiment \#2 (fixed discrimination capacity $\geq .70$ and $\leq .80$ ). (a) Relationship between the maximum abundance value in the testing dataset $(\max (N))$ and strength of the wedge-shaped pattern $(D)$ considering all cases. In (b), the relationship between $\max (N)$ and $D$ is shown only for cases with prevalence $\leq .1$; in (c), for cases with prevalence in $(.1, .2]$; in (d), for cases with prevalence in $(.2, .3]$; in (e), for cases with prevalence in $(.3, .4]$; in (f), for cases with prevalence in $(.4, .5]$; in $(\mathrm{g})$, for cases with prevalence in $(.5, .6]$; in $(\mathrm{h})$, for cases with prevalence in $(.6, .7]$; in (i), for cases with prevalence in $(.7, .8]$; in (j), for cases with prevalence in $(.8, .9]$; in $(\mathrm{k})$, for cases with prevalence $>.9$. The red line corresponds to the local polynomial regression fit (loess function in $\mathrm{R}$ with 1-degree polynomial and span $=0.25$ ). (1) Surface plot of $D$ as a function of $\max (N)$ and prevalence. Warm colour indicates $D>0$, cool colour indicates $D<0$, white colour indicates $D=0$. Red contour line corresponds to the limit of $D=0$ 
Our results show that as maximum abundance increases, the effect of SDM performance becomes more apparent. Thus, in general, as discrimination capacity rises, $D$ tends to decrease while $R$ shows the opposite trend, indicating that the capacity of suitability to predict abundance improves as the discrimination power of the SDMs becomes better, as other authors have suggested (e.g. Jiménez-Valverde, 2011). This makes sense: if a model cannot discriminate between presence and absence cases, it cannot be expected to predict abundance in any way; only when the model starts to yield accurate predictions, can it be expected to start accounting-at least to some extent - for abundance. Figure 4 shows a sequence of abundance-suitability relationships from poorly to highly discriminative SDMs (all of them carried out at the same prevalence of .5). In the abundance-suitability graph, as the discrimination power of the SDMs increases, absence data cases shift towards the bottom lefthand corner while presence data cases shift towards the right-hand side. $D$ is maximum $(D=219.16)$ and $R$ is minimum $(R=.02)$ when discrimination capacity is lower $\left(S e^{*}=.60\right.$; Figure $\left.4 \mathrm{a}\right)$, while the opposite happens $(D=4.75$ and $R=.85)$ when discrimination capacity is higher $\left(S e^{*}=.95\right.$; Figure $\left.4 \mathrm{c}\right)$. Figure 4 also shows that, despite previous theoretical and empirical support (Carrascal et al., 2015; Gerrard \& Chiang, 1970; Gerrard \& Cook, 1972), a linear model might not be the best way to characterize the abundance-suitability relationship (e.g. VanDerWal et al., 2009). However, the application of nonlinear models might be problematic too (see Jiménez-Valverde, 2011), and in our opinion, the approach followed in the present study is a straightforward and enlightening way to measure the strength of the wedge-shaped relationship, as shown by the clear and consistent patterns found. Moving on, despite the overall trend found between $D$ and discrimination capacity, $D$ shows an important amount of variation along the discrimination gradient; to fully understand the results, it is necessary to take into account species prevalence.

The negative relationship between $D$ and discrimination power is consistent for a prevalence range between .2 and .7, approximately. However, $D$ shows an important amount of variance which is not homogeneous along the discrimination gradient, decreasing as long as discrimination increases. We believe that stochasticity might play a role here, such that wedge-shaped patterns can arise or not depending on the exact disposition of the points over the abundance-suitability graph space. The lower the discrimination power, the higher is the effect of stochasticity on $D$, whereas the goodness-of-fit of the quantile models shows a consistent positive and more or less linear trend with discrimination. In the case of very low prevalence values, the effect of stochasticity on $D$ arises when discrimination capacity is high, because the exact position of the few presence cases on the right-hand side of the abundance-suitability graph heavily conditions the regression slope (Figure 4). The possibility for stochasticity to give rise to high $D$ values decreases as long as the number of absence cases decreases thus reducing their dominance over the regression slope. In fact, when prevalence is over .8, D is usually close to zero or even negative. However, in the case of very common species, there is an important 
drop in $R$, and even highly discriminative scenarios can show low $R$ values, indicating the inability of these SDMs to predict local abundance. Taken to an extreme, we invite the reader to imagine a 'perfect' SDM able to completely discriminate presences from absences, with all absence cases located on the bottom left-hand corner of the abundance-suitability graph while all presence cases are located in a vertical line on the right-hand side (Figure 4). Such a perfect model would only correctly predict zero abundance cases, but if the prevalence was very high, the amount of zero abundance cases would be very low. Thus, the paradox is that a perfect discriminative SDM would be completely unable to predict abundance, especially when the species prevalence was high. The fact that $R$ values are always lower than 1 means that suitability can only predict the maximum value of abundance to some extent, something that seems to be unavoidable as discrimination capacity increases (see Jiménez-Valverde, 2011).

(a)
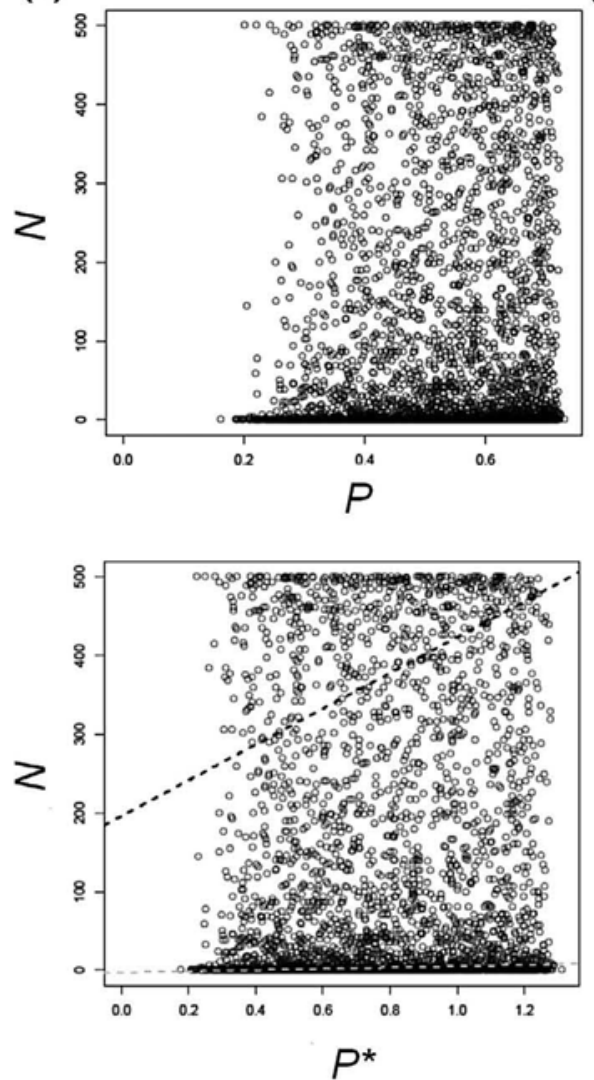

(b)
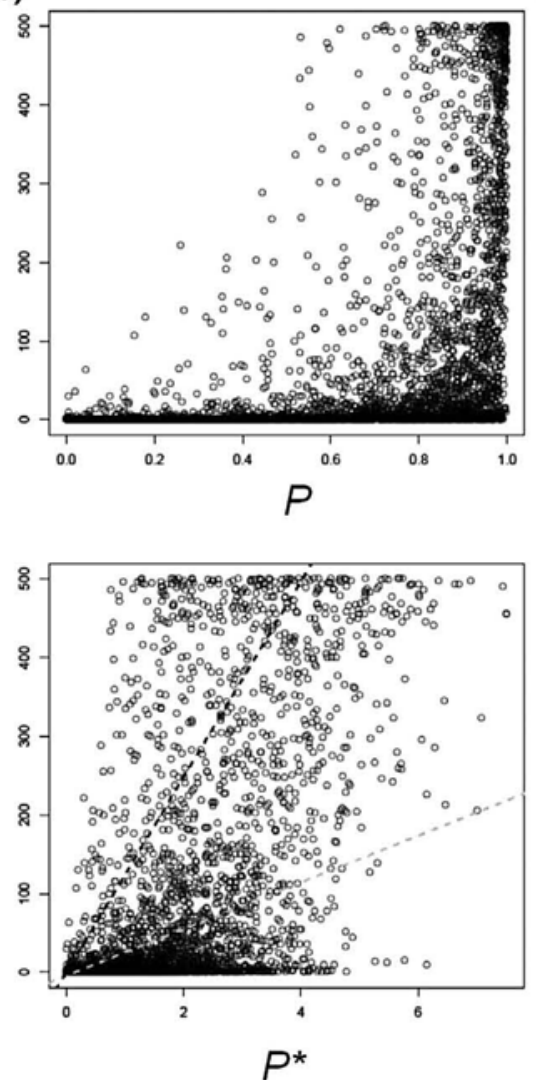

(c)
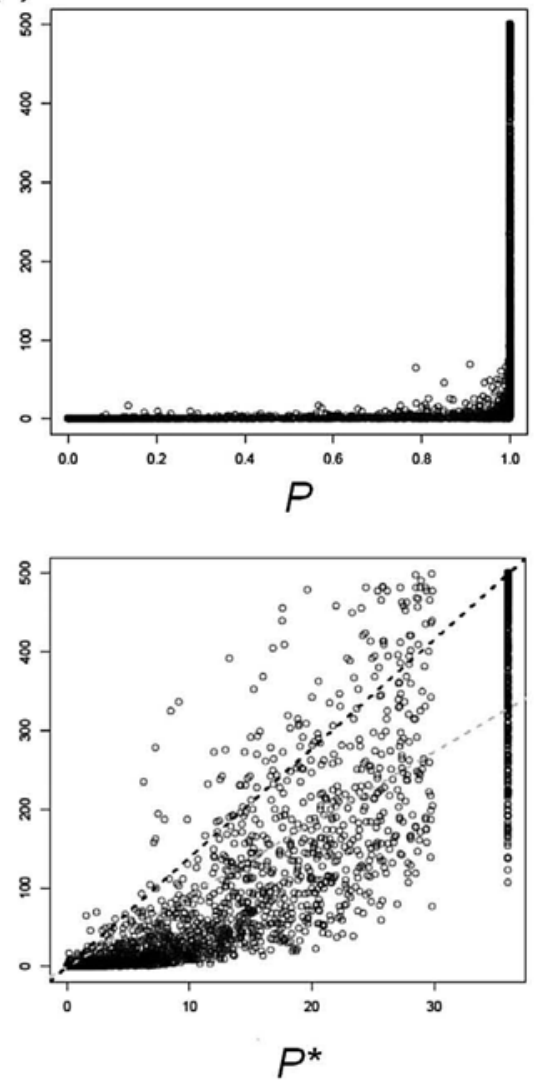

FIGURE 4 Abundance versus suitability plots (top, $N$ versus $P$; bottom, $N$ versus $P^{*}$ ) for three different cases in experiment \#1. Prevalence equals .5 in the three cases. Dashed lines are the 50th (in grey) and 90th (black) quantile regression lines. (a) Discrimination capacity $\left(S e^{*}\right)=.60, R=.02$, strength of the wedge-shaped pattern $(D)=219.16$. (b) $S e^{*}=.83, R=.50, D=94.54$. (c) $S e^{*}=.95, R=.85, D=4.75$. Although $P^{*}$ values were used in the analyses (see argumentation in section 2.4 of the main text), in this figure we also show the corresponding $N$ versus $P$ plots in the upper panels to better reflect how the points move across the graph space as discrimination capacity increases (see Discussion), as well as to visualize the effect of transforming $P$ into $P^{*}$ 
Our results show unequivocally that information loss exerts a clear effect when converting abundance into presence-absence. Information loss, which means that a presence can correspond to a wide range of abundance values, renders the relationship between suitability and abundance a textbook example of unavoidable heteroscedasticity. Wedge-shaped patterns are expected to be the norm rather than the exception, although their strength is determined by the discrimination capacity of the SDMs and by prevalence. Prevalence, together with maximum abundance, exerts an important effect on $D$ by conditioning the amount of information that can be lost when converting abundance into presence-absence data. Consequently, prevalence must be taken into account when assessing the strength of the wedge-shaped pattern and when doing comparative studies.

Although unavoidably related, occurrence and abundance are not the same variable. In fact, changes in abundance can occur without altering the occurrence of the species (Ashcroft et al., 2017). Thus, the relationship between suitability and abundance will mostly, and inevitably, be wedge-shaped and SDMs cannot be expected to predict actual local abundance, but only its relative potential at best, even when the variables influencing abundance are considered. More generally, wedge-shaped patterns are the consequence of the heteroscedasticity derived from information loss when transforming abundance into presence-absence. In real case scenarios, different processes acting at several spatial scales may cause abundance and suitability to decouple and, consequently, add heteroscedasticity in their relationship. For example, abundance may respond to factors that significantly vary over microscales, competitive exclusion may push individuals to unfavourable areas, and source-sink and other dispersal dynamics may add noise to the environmental determinism of the species (Bradley, 2016; Dallas \& Hastings, 2018; Gaston, 2003; Osorio-Olvera et al., 2019; Van Horne, 1983; Weber et al., 2016). In addition, different sampling schemes with varied underlying detectability functions (e.g. data from GBIF versus data from a standardized survey) may also add complexity to the abundance-suitability relationship. Only in the hypothetical case of the probability of detection being directly proportional to local abundance, and the factors determining abundance being completely known, could the suitability derived from a well-calibrated SDM (see Jiménez-Valverde et al., 2013) be expected to perfectly predict abundance, because in this case suitability and abundance would be virtually the same variable. Previously, Jiménez-Valverde (2011) reached a similar conclusion and warned of possible circularity in the abundance-suitability relationship. However, the conditions for this to happen are unrealistic under real working situations, especially when considering broad geographical and temporal scales. Finally, in our virtual species, the prevalence of both the training and testing samples equalled the prevalence of the species, as would be expected in a standardized survey. However, the greater the difference between the species and the sampling prevalence, the higher the uncertainty in the abundance-suitability relationship. Understanding that a wedge-shaped pattern is essentially caused by unavoidable heteroscedasticity due to information loss, and that it is controlled by the performance 
capacity of the SDMs and by the prevalence of the samples, is fundamental to address and interpret future studies exploring the abundance-suitability relationship in greater depth.

The conclusion is that prediction of abundance should not be the objective of SDMs that are built from presence-absence data. Continuous suitability values derived from SDMs fed by occurrence data may often transmit a false sense of precision (Hirzel \& Le Lay, 2008), and these maps should not be used in isolation but at best as a complementary procedure when the purpose is to assist in conservation decision-making requiring species abundance or persistence information. This reinforces the idea of using SDMs as a qualitative rather than a quantitative means of assessment (Jiménez-Valverde, 2014).

\section{ACKNOWLEDGMENTS}

This paper is a contribution to the projects NICHEAPPS (CGL2014-56416-P) and DistriMod (CGL2017-89000-P) led by P.A. and A.J.-V., respectively, and funded by the Spanish Ministry of Economy and Competitiveness (MINECO, now Ministry of Science and Innovation). A.J.-V. and P.A. were supported by the MINECO (now Ministry of Science and Innovation) Ramón y Cajal Programme (RYC-2013-14441 and RYC-2011-07670, respectively). We are grateful to Luis M. Carrascal for extensive discussions on the topic.

\section{DATA AVAILABILITY STATEMENT}

This study is entirely based on simulations; no new data have been used. The R code and necessary files for running the simulations are provided in Supporting Information Appendix S2.

\section{REFERENCES}

Acevedo, P., Ferreres, J., Escudero, M. A., Jiménez, J., Boadella, M., \& Marco, J. (2017). Population dynamics affect the capacity of species distribution models to predict species abundance on a local scale. Diversity and Distributions, 23, 1008-1017. https://doi.org/10.1111/ddi.12589

Acevedo, P., Jiménez-Valverde, A., Aragón, P., \& Niamir, A. (2016). New developments in the study of species distribution. In J. T. Garcia, R. Mateo, \& B. Arroyo (Eds.), Current trends in wildlife research (pp. 151-175). Wildlife Research Monographs. Springer.

Ashcroft, M. B., King, D. H., Raymond, B., Turnbull, J. D., Wasley, J., \& Robinson, S. A. (2017). Moving beyond presence and absence when examining changes in species distributions. Global Change Biology, 23, 2929-2940. https://doi.org/10.1111/gcb.13628

Bahn, V., \& McGill, B. J. (2013). Testing the predictive performance of distribution models. Oikos, 122, 321-331. https://doi. org/10.1111/j.1600-0706.2012.00299.x 
Bradley, B. A. (2016). Predicting abundance with presence-only models. Landscape Ecology, 31, 19-30. https://doi.org/10.1007/s1098 0-015-0303-4

Brown, J. H., Mehlman, D. W., \& Stevens, G. C. (1995). Spatial variation in abundance. Ecology, 76, 2028-2043. https://doi.org/10.2307/ 1941678

Brown, J. H., Stevens, G. C., \& Kaufman, D. M. (1996). The geographic range: Size, shape, boundaries, and internal structure. Annual Review of Ecology and Systematics, 27, 597-623. https://doi.org/10.1146/

Cade, B. S., \& Noon, B. R. (2003). A gentle introduction to quantile regression for ecologists. Frontiers in Ecology and the Environment, 1, 412-420. https://doi.org/10.1890/1540-9295(2003)001[0412:AGITQ $\mathrm{R}] 2.0 . \mathrm{CO} ; 2$

Carrascal, L. M., Aragón, P., Palomino, D., \& Lobo, J. M. (2015). Predicting regional densities from bird occurrence data: Validation and effects of species traits in a Macaronesian Island. Diversity and Distributions, 21, 1284-1294. https://doi.org/10.1111/ddi.12368

Carrascal, L. M., Moreno, A. C., Delgado, A., Suárez, V., \& Trujillo, D. (2017). Habitat suitability - density relationship in an endangered woodland species: The case of the blue chaffinch (Fringilla polatzeki). PeerJ, 5, e3771.

Dallas, T., Decker, R. R., \& Hastings, A. (2017). Species are not most abundant in the centre of their geographic range or climatic niche. Ecology Letters, 20, 1526-1533. https://doi.org/10.1111/ele.12860

Dallas, T. A., \& Hastings, A. (2018). Habitat suitability estimated by niche models is largely unrelated to species abundance. Global Ecology and Biogeography, 27, 1448-1456. https://doi.org/10.1111/ geb. 12820

Ehrlén, J., \& Morris, W. F. (2015). Predicting changes in the distribution and abundance of species under environmental change. Ecology Letters, 18, 303-314. https://doi.org/10.1111/ele.12410

Franklin, J. (2009). Mapping species distributions. Spatial inference and prediction. Cambridge University Press.

Fukuda, S., \& De Baets, B. (2016). Data prevalence matters when assessing species' responses using data-driven species distribution models. Ecological Informatics, 32, 69-78. https://doi.org/10.1016/j. ecoinf.2016.01.005

Gaston, K. J. (2003). The structure and dynamics of geographic ranges. Oxford University Press.

Gerrard, D. J., \& Chiang, H. C. (1970). Density estimation of corn rootworm egg populations based upon frequency of occurrence. Ecology, 51, 235-243. https://doi.org/10.2307/1933659

Gerrard, D. J., \& Cook, R. D. (1972). Inverse binomial sampling as a basis for estimating negative binomial population densities. Biometrics, 28, 971-980. https://doi.org/10.2307/2528633 
Gräler, B., Pebesma, E., \& Heuvelink, G. (2016). Spatio-temporal interpolation using gstat. The R Journal, 8, 204-218. https://doi. org/10.32614/RJ-2016-014

Grinnell, J. (1917). The niche-relationships of the California thrasher. The Auk, 34, 427-433. https://doi.org/10.2307/4072271

Guarino, E. S. G., Barbosa, A. M., \& Waechter, J. L. (2012). Occurrence and abundance models of threatened plant species: Applications to mitigate the impact of hydroelectric power dams. Ecological Modelling, 230, 22-33. https://doi.org/10.1016/j.ecolmodel.2012.01.007

Guisan, A., Thuiller, W., \& Zimmermann, N. E. (2017). Habitat suitability and distribution models with applications in R. Cambridge University Press.

Guisan, A., Tingley, R., Baumgartner, J. B., Naujokaitis-Lewis, I., Sutcliffe, P. R., Tulloch, A. I. T., Regan, T. J., Brotons, L., McDonald-Madden, E., Mantyka-Pringle, C., Martin, T. G., Rhodes, J. R., Maggini, R., Setterfield, S. A., Elith, J., Schwartz, M. W., Wintle, B. A., Broennimann, O., Austin, M., ... Buckley, Y. M. (2013). Predicting species distributions for conservation decisions. Ecology Letters, 16, 1424-1435. https://doi.org/10.1111/ele.12189

Gutiérrez, D., Harcourt, J., Díez, S. B., Gutiérrez Illán, J., \& Wilson, R.J. (2013). Models of presence-absence estimate abundance as well as (or even better than) models of abundance: The case of the butterfly Parnassius apollo. Landscape Ecology, 28, 401-413. https://doi. org/10.1007/s10980-013-9847-3

Hengeveld, R. (1990). Dynamic biogeography. Cambridge University Press.

Hirzel, A. H., \& Le Lay, G. (2008). Habitat suitability modelling and niche theory. Journal of Applied Ecology, 45, 1372-1381. https://doi. org/10.1111/j.1365-2664.2008.01524.x

Hutchinson, G. E. (1957). Concluding remarks. Cold Spring Harbor Symposia on Quantitative Biology, 22, 415-427. https://doi.org/10.1101/ SQB.1957.022.01.039

Hutchinson, G. E. (1978). An introduction to population ecology. Yale University Press.

Jiménez-Valverde, A. (2011). Relationship between local population density and environmental suitability estimated from occurrence data. Frontiers of Biogeography, 3, 59-61.

Jiménez-Valverde, A. (2012). Insights into the area under the receiver operating characteristic curve as a discrimination measure in species distribution modelling. Global Ecology and Biogeography, 21, 498-507.

Jiménez-Valverde, A. (2014). Threshold-dependence as a desirable attribute for discrimination assessment: Implication for species distribution modelling. Biodiversity and Conservation, 23, 369-385.

Jiménez-Valverde, A. (2020). Sample size for the evaluation of presence-absence models. Ecological Indicators, 114, 106289. 
Jiménez-Valverde, A., Acevedo, P., Barbosa, A. M., Lobo, J. M., \& Real, R. (2013). Discrimination capacity in species distribution models depends on the representativeness of the environmental domain. Global Ecology and Biogeography, 22, 508-516. https://doi.org/10.1111/geb.12007

Jiménez-Valverde, A., Diniz, F., Azevedo, E. B., \& Borges, P. A. V. (2009). Species distribution models do not account for abundance: The case of arthropods on Terceira Island. Annales Zoologici Fennici, 46, 451-464. https://doi.org/10.5735/086.046.0606

Jiménez-Valverde, A., Lira-Noriega, A., Peterson, A. T., \& Soberón, S. (2010). Marshalling existing biodiversity data to evaluate biodiversity status and trends in planning exercises. Ecological Research, 25, 947-957. https://doi.org/10.1007/s11284-010-0753-8

Jiménez-Valverde, A., Lobo, J. M., \& Hortal, J. (2009). The effect of prevalence and its interaction with sample size on the reliability of species distribution models. Community Ecology, 10, 196-205. https:// doi.org/10.1556/ComEc.10.2009.2.9

Jiménez-Valverde, A., Peterson, A. T., Soberón, J., Overton, J., Aragón, P., \& Lobo, J. M. (2011). Use of niche models in invasive species risk assessments. Biological Invasions, 13, 2785-2797. https://doi. org/10.1007/s10530-011-9963-4

Kim, Y. J., \& Gu, C. (2004). Smoothing spline gaussian regression: More scalable computation via efficient approximation. Journal of the Royal Statistical Society B, 66, 337-356. https://doi.org/10.1046/ j.1369-7412.2003.05316.x

Kissling, W. D., Ahumada, J. A., Bowser, A., Fernandez, M., Fernández, N., García, E. A., Guralnick, R. P., Isaac, N. J. B., Kelling, S., Los, W., McRae, L., Mihoub, J.-B., Obst, M., Santamaria, M., Skidmore, A. K., Williams, K. J., Agosti, D., Amariles, D., Arvanitidis, C., ... Hardisty, A. R. (2018). Building essential biodiversity variables (EBVs) of species distribution and abundance at a global scale. Biological Reviews, 93, 600-625. https://doi.org/10.1111/brv.12359

Koenker, R. (2018).quantreg: Quantile regression. R package version 5.36. http://CRAN.R-project.org/package=quantreg

Koenker, R., \& Machado, J. (1999). Goodness of fit and related inference processes for quantile regression. Journal of the American Statistical Association, 94, 1296-1310. https://doi.org/10.1080/01621459.1999. 10473882

Kunin, W. E. (1998). Extrapolating species abundance across spatial scales. Science, 281, 1513-1515. https://doi.org/10.1126/scien ce.281.5382.1513

Lobo, J. M., Jiménez-Valverde, A., \& Real, R. (2008). AUC: A misleading measure of the performance of predictive distribution models. Global Ecology and Biogeography, 17, 145-151. https://doi. 
$\operatorname{org} / 10.1111 / j .1466-8238.2007 .00358 . x$

Lowe, W. H., \& McPeek, M. A. (2014). Is dispersal neutral? Trends in Ecology and Evolution, 29, 444-450. https://doi.org/10.1016/j. tree.2014.05.009

Lunghi, E., Manenti, R., Mulargia, M., Veith, M., Corti, C., \& Ficetola, G. F. (2018). Environmental suitability models predict population density, performance and body condition for microendemic salamanders. Scientific Reports, 8, 7527. https://doi.org/10.1038/s41598-01825704-1

Martínez-Meyer, E., Díaz-Porras, D., Peterson, A. T., \& Yañez-Arenas, C. (2012). Ecological niche structure and range wide abundance patterns of species. Biology Letters, 9, 20120637.

Muñoz, A. R., Jiménez-Valverde, A., Márquez, A. L., Moleón, M., \& Real, R. (2015). Environmental favourability as a cost-efficient tool to estimate carrying capacity. Diversity and Distributions, 21, 1388-1400. https://doi.org/10.1111/ddi.12352

Nielsen, S. E., Johnson, C. J., Heard, D. C., \& Boyce, M. S. (2005). Can models of presence-absence be used to scale abundance? Two case studies considering extremes in life history. Ecography, 28, 197-208. https://doi.org/10.1111/j.0906-7590.2005.04002.x

O'Grady, J. J., Reed, D. H., Brook, B. W., \& Frankham, R. (2004). What are the best correlates of predicted extinction risk? Biological Conservation, 118, 513-520. https://doi.org/10.1016/j. biocon.2003.10.002

Osorio-Olvera, L., Soberón, J., \& Falconi, M. (2019). On population abundance and niche structure. Ecography, 42, 1415-1425. https://doi. org/10.1111/ecog.04442

Osorio-Olvera, L., Yañez-Arenas, C., Martínez-Meyer, E., \& Peterson, A.T. (2020). Relationships between population densities and niche-centroid distances in North American birds. Ecology Letters, 23, 555-564. https://doi.org/10.1111/ele.13453

Parham, P. E., Waldock, J., Christophides, G. K., Hemming, D., Agusto, F., Evans, K. J., Fefferman, N., Gaff, H., Gumel, A., LaDeau, S., Lenhart, S., Mickens, R. E., Naumova, E. N., Ostfeld, R. S., Ready, P. D., Thomas, M. B., Velasco-Hernandez, J., \& Michael, E. (2015). Climate, environmental and socio-economic change: Weighing up the balance in vector-borne disease transmission. Philosophical Transactions of the Royal Society B: Biological Sciences, 370, 20130551. https://doi. org/10.1098/rstb.2013.0551

Parker, I. M., Simberloff, D., Lonsdale, W. M., Goodell, K., Wonham, M., Kareiva, P. M., \& Goldwasser, L. (1999). Impact: Toward a framework for understanding the ecological effects of invaders. Biological Invasions, 1, 3-19.

Pearce, J., \& Ferrier, S. (2001). The practical value of modelling relative abundance of species for regional conservation planning: A case study. Biological Conservation, 98, 33-43. https://doi.org/10.1016/ 


\section{S0006-3207(00)00139-7}

Peterson, A. T., Soberón, J., Pearson, R. G., Anderson, R. P., Martínez-Meyer, E., Nakamura, M., \& Araújo, M. B. (2011). Ecological niches and geographic distributions. Princeton University Press.

Phillips, S. J., Anderson, R. P., \& Schapire, R. E. (2006). Maximum entropy modeling of species geographic distributions. Ecological Modelling, 190, 231-259. https://doi.org/10.1016/j.ecolmodel.2005.03.026

Pimm, S. L., Jones, H. L., \& Diamond, J. (1988). On the risk of extinction. The American Naturalist, 132, 757-785. https://doi.org/10.1086/284889

R Development Core Team. (2014). R: A language and environment for statistical computing. R Foundation for Statistical Computing. http:// www.R-project.org

Real, R., Barbosa, A. M., \& Vargas, J. M. (2006). Obtaining environmental favourability functions from logistic regression. Environmental and Ecological Statistics, 13, 237-245. https://doi.org/10.1007/s1065 $1-005-0003-3$

Salguero-Gómez, R., Jones, O. R., Archer, C. R., Bein, C., de Buhr, H., Farack, C., Gottschalk, F., Hartmann, A., Henning, A., Hoppe, G., Römer, G., Ruoff, T., Sommer, V., Wille, J., Voigt, J., Zeh, S., Vieregg, D., Buckley, Y. M., Che-Castaldo, J., ... Vaupel, J. W. (2016). COMADRE: A global data base of animal demography. Journal of Animal Ecology, 85, 371-384. https://doi.org/10.1111/1365-2656.12482

Santika, T. (2011). Assessing the effect of prevalence on the predictive performance of species distribution models using simulated data. Global Ecology and Biogeography, 20, 181-192. https://doi. org/10.1111/j.1466-8238.2010.00581.x

Santini, L., Isaac, N. J. B., \& Ficetola, G. F. (2018). TetraDENSITY: A data-base of population density estimates in terrestrial vertebrates. Global Ecology and Biogeography, 27, 787-791. https://doi.org/10.1111/ geb.12756

Sing, T., Sander, O., Beerenwinkel, N., \& Lengauer, T. (2005). ROCR: Visualizing classifier performance in R. Bioinformatics, 21, 3940-3941. https://doi.org/10.1093/bioinformatics/bti623

Soberón, J. (2007). Grinnellian and Eltonian niches and geographic distributions of species. Ecology Letters, 10, 1115-1123. https://doi. org/10.1111/j.1461-0248.2007.01107.x

Soininen, J., \& Heino, J. (2005). Relationships between local population persistence, local abundance and regional occupancy of species: Distribution patterns of diatoms in boreal streams. Journal of Biogeography, 32, 1971-1978. https://doi.org/10.1111/j.13652699.2005.01342.x

Somodi, I., Lepesi, N., \& Botta-Dukátet, Z. (2017). Prevalence, statistical thresholds, and accuracy assessment for species distribution models. Ecology and Evolution, 7, 863-872.

Thibault, K. M., Supp, S. R., Giffin, M., White, E. P., \& Ernest, S. (2011). Species composition and abundance 
of mammalian communities. Ecology, 92, 2316. https://doi.org/10.1890/11-0262.1

Thomson, J., Weiblen, G., Thomson, B., Alfaro, S., \& Legendre, P. (1996). Untangling multiple factors in spatial distributions: Lilies, gophers, and rocks. Ecology, 77, 1698-1715. https://doi.org/10.2307/ 2265776

Tôrres, N. M., De Marco, P., Santos, T., Silveira, L., de Almeida Jácomo,A. T., \& Diniz-Filho, J. A. F. (2012). Can species distribution modelling provide estimates of population densities? A case study with jaguars in the Neotropics. Diversity and Distributions, 18, 615-627. https:// doi.org/10.1111/j.1472-4642.2012.00892.x

Van Horne, B. (1983). Density as a misleading indicator of habitat quality. Journal of Wildlife Management, 47, 893-901. https://doi. org/10.2307/3808148

VanDerWal, J., Shoo, L. P., Johnson, C. N., \& Williams, S. E. (2009). Abundance and the environmental niche: Environmental suitability estimated from niche models predicts the upper limit of local abundance. The American Naturalist, 174, 282-291. https://doi. org/10.1086/600087

Weber, M. M., Stevens, R. D., Diniz-Filho, J. A. F., \& Grelle, C. E. V. (2016). Is there a correlation between abundance and environmental suitability derived from ecological niche modelling? A meta-analysis. Ecography, 39, 1-12.

Williams, P. H., \& Araújo, M. B. (2000). Using probability of persistence to identify important areas for biodiversity conservation. Proceedings of the Royal Society B: Biological Sciences, 267, 1959-1966. https://doi. org/10.1098/rspb.2000.1236

Wood, S. N. (2003). Thin-plate regression splines. Journal of the Royal Statistical Society B, 65, $95-114$. https://doi.org/10.1111/14679868.00374

Wood, S. N. (2004). Stable and efficient multiple smoothing parameter estimation for generalized additive models. Journal of the American Statistical Association, 99, 673-686. https://doi.org/10.1198/01621 4504000000980

Wood, S. N. (2017). Generalized additive models: An introduction with R. Chapman and Hall/CRC Press.

Yin, D., \& He, F. (2014). A simple method for estimating species abundance from occurrence maps. Methods in Ecology and Evolution, 5, 336-343. https://doi.org/10.1111/2041-210X.12159

\section{SUPPORTING INFORMATION}

Additional supporting information may be found online in the Supporting Information section. 